\title{
Multimodal validation of focal enhancement in intracranial aneurysms as a surrogate marker for aneurysm instability
}

\author{
Naomi Larsen ${ }^{1}$ (D) . Charlotte Flüh ${ }^{2}$ (D) Sylvia Saalfeld ${ }^{3,4}$ (D) Samuel Vo $ß^{3,5}$ (D) Georg Hille ${ }^{3,4} \cdot$ David Trick $^{6}$. \\ Fritz Wodarg $^{1}$ (D) Michael Synowitz ${ }^{2}$ (D) Olav Jansen $^{1}$ (D) $\cdot$ Philipp Berg M $^{3,5}$
}

Received: 1 May 2020 / Accepted: 13 July 2020 / Published online: 17 July 2020

(C) The Author(s) 2020

\begin{abstract}
Purpose Circumferential enhancement on MR vessel wall imaging has been proposed as a biomarker of a higher risk of rupture in intracranial aneurysms. Focal enhancement is frequently encountered in unruptured aneurysms, but its implication for risk stratification and patient management remains unclear. This study investigates the association of focal wall enhancement with hemodynamic and morphological risk factors and histologic markers of wall inflammation and degeneration.

Methods Patients with an unruptured middle cerebral artery aneurysm who underwent 3D rotational angiography and 3T MR vessel wall imaging showing focal wall enhancement were included. Hemodynamic parameters were calculated based on flow simulations and compared between enhanced regions and the entire aneurysm surface. Morphological parameters were semiautomatically extracted and quantitatively associated with wall enhancement. Histological analysis included detection of vasa vasorum, CD34, and myeloperoxidase staining in a subset of patients.

Results Twenty-two aneurysms were analyzed. Enhanced regions were significantly associated with lower AWSS, lower maxOSI, and increased LSA. In multivariate analysis, higher ellipticity index was an independent predictor of wall enhancement. Histologic signs of inflammation and degeneration and higher PHASES score were significantly associated with focal enhancement.

Conclusion Focal wall enhancement is colocalized with hemodynamic factors that have been related to a higher rupture risk. It is correlated with morphological factors linked to rupture risk, higher PHASES score, and histologic markers of wall destabilization. The results support the hypothesis that focal enhancement could serve as a surrogate marker for aneurysm instability.
\end{abstract}

Keywords Intracranial aneurysm $\cdot$ Subarachnoid hemorrhage $\cdot$ Magnetic resonance imaging $\cdot$ Hemodynamics $\cdot$ Morphology Histology

Naomi Larsen and Charlotte Flüh contributed equally to this work.

Naomi Larsen

naomi.larsen@uksh.de

1 Department of Radiology and Neuroradiology, University Hospital Schleswig-Holstein Campus Kiel, Arnold-Heller-Str. 3, Haus D, 24105 Kiel, Germany

2 Department of Neurosurgery, University Hospital Schleswig-Holstein, Campus Kiel, Kiel, Germany

3 Forschungscampus STIMULATE, University of Magdeburg, Magdeburg, Germany

4 Department of Simulation and Graphics, University of Magdeburg, Magdeburg, Germany

5 Institute of Fluid Dynamics and Thermodynamics, University of Magdeburg, Magdeburg, Germany

6 Institute of Pathology, University Hospital Schleswig-Holstein, Campus Kiel, Kiel, Germany

\section{Introduction}

Intracranial aneurysms have a prevalence of up to $3 \%$ and generally harbor a low risk of rupture $[1,2]$. Still, subarachnoid hemorrhage following rupture of an intradural aneurysm is associated with considerable mortality and morbidity [3]. Therefore, risk stratification of patients diagnosed with an unruptured intradural aneurysm is crucial, but optimal management remains controversial. Recently, published data suggest an association of wall enhancement in intracranial saccular aneurysms on MR vessel wall imaging with a higher risk for rupture [4-7]. Specifically, thick circumferential wall enhancement has been linked to a higher risk of rupture [4]. Moreover, wall enhancement probably identifies ruptured aneurysms in patients with subarachnoid hemorrhage and multiple aneurysms [8], and in AVMs, respectively [9]. In a prior study, inflammatory and degenerative wall changes were 
detected in a histologic analysis of unruptured aneurysms showing wall enhancement [10].

Focal enhancement has been reported to be associated with ruptured aneurysms and the rupture site $[11,12]$. While low-flow conditions near the aneurysm wall have been described to coincide with focal enhancement [13], low wall shear stress (WSS) has been found to be associated with rupture sites [14]. Other studies related flow characteristics, such as low shear area (LSA), shear concentration index (SCI), oscillatory shear index (OSI), neck inflow rate (NIR), inflow concentration index (ICI), and WSS to aneurysm growth, rupture risk, and rupture [15-21].

Moreover, numerous morphological parameters including size ratio, undulation index, nonsphericity index, ellipticity index, aneurysm angle, aneurysm volume, neck size. and aspect ratio have been associated with aneurysm rupture [22-24].

Focal enhancement in unruptured aneurysms is frequently encountered on MR vessel wall imaging; still, its implication for patient risk stratification and management remains unclear. Hence, the goal of this study was to determine the correlation of focal enhancement with previously described risk factors in order to assess its suitability as a surrogate marker for a higher risk of rupture. To achieve this goal, we chose a multimodal approach: We investigated whether focal wall enhancement is colocalized with specific hemodynamic conditions related to a higher risk of rupture. Furthermore, the association with morphological parameters linked to rupture risk was assessed. Histologic data were analyzed to associate pathologic processes encountered during aneurysm evolution and wall remodeling with wall enhancement.

\section{Methods}

\section{Patients}

Ethics approval for this study was obtained from the local ethics committee. Written informed consent was obtained. We selected all patients from our institutional MR vessel wall imaging database with an unruptured middle cerebral artery aneurysm, who received high-resolution 3T MR vessel wall imaging showing focal wall enhancement and threedimensional rotational angiography (3D-RA) from 7/2016 to 3/2019. Only cases with MRI and 3D-RA images of sufficient quality were included. Demographic data as age, gender, intake of acetylsalicylic acid and nicotine, hypertension, diabetes, and PHASES score were extracted from patient records. Histologic data were collected from a subset of patients who underwent microsurgical clipping of the aneurysm. In our institution, the neurosurgeon and interventional neuroradiologist determine in an interdisciplinary consensus-based decision-making process whether to recommend treatment of the aneurysm and the preferred method.

\section{MR vessel wall imaging}

MR vessel wall imaging was acquired on a $3 T$ MR scanner (Achieva, Philips Healthcare, Best, The Netherlands) equipped with a 32-channel head coil. The protocol comprised a T1-weighted black blood 3D variable refocusing flip angle sequence (VISTA) (TE/TR, 27/700 ms; matrix, 268 ×332; field of view, $200 \times 250 \times 160 \mathrm{~mm}$; voxel size, $0.75 \times$ $0.75 \times 0.75 \mathrm{~mm}$; acquisition time, $4 \min 45 \mathrm{~s}$ ) and a TOFangiography; both sequences were acquired before and after administration of $0.1 \mathrm{mmol} / \mathrm{kg}$ gadoterate meglumine (Dotarem, Guerbet, Villepinte, France).

Contrast enhancement of the aneurysm wall was visually assessed on multiplanar reformatted postcontrast 3D T1 black blood images.

Focal wall enhancement was defined as a hyperintense signal adjacent to the black blood lumen or wall-adherent thrombus with at least one clearly discernible unenhanced wall segment of any extent. The presence of a double-layer appearance indicative of thrombus, defined as a non-enhancing, walladjacent structure bordered by an enhancing layer on both the luminal and the wall-adjacent surface, was documented.

Two neuroradiologists (N.L., F.W.), each with at least 9 years of experience in neurovascular imaging, who were blinded for clinical and hemodynamic data, independently assessed the aneurysm wall. Disagreement between the readers was solved by consensus.

\section{Digital subtraction angiography}

Digital subtraction angiography (DSA) was performed under local anesthesia on a biplane flat panel DSA unit (Allura Xper FD 20/10, Philips, Best, The Netherlands) via a femoral access. 3D-RA was performed with a selective contrast injection of $20 \mathrm{ml}$ Imeron 300 (Iomeprol, Bracco Imaging, Milan, Italy) in the ipsilateral internal carotid artery at a flow rate of $2 \mathrm{ml} / \mathrm{s}$. The dataset was transferred to a dedicated workstation (XtraVision, Philips, Best, The Netherlands) and reconstructed with a voxel size of $0.27 \times 0.27 \times 0.27 \mathrm{~mm}^{3}$.

\section{Vessel segmentation and image co-registration}

Segmentation of vessel surfaces from 3D-RA datasets and coregistration to MRI was carried out using MeVisLab 2.7 (MeVis Medical Solutions AG, Bremen, Germany). A threshold-based segmentation approach was applied to the 3D-RA images, followed by marching cubes to obtain a triangulated surface mesh. Enhanced regions were manually delineated in black blood MR images using the contour segmentation objects library of MeVisLab. The enhancement mask was converted into a triangle surface mesh as well. Finally, coregistration utilizing a rigid multi-resolution registration [25] with a Quasi-Newton optimizer within MeVisLab yielded 
transformation matrices for each patient's dataset. Applying the transformation matrices to the manually segmented enhancement regions allowed for the combination with the vessel surface (see Fig. 1). The percentage of the aneurysm surface area covered by enhanced regions was determined for each aneurysm (enhancement area EA) and utilized as a quantitative measure for the extent of enhancement.

\section{Hemodynamic simulations}

Based on the segmented aneurysm surfaces, hemodynamic simulations derived from 3D-RA datasets were carried out in all cases using STAR-CCM+ 13.04 (Siemens PLM Software Inc., Plano, TX, USA). For spatial discretization a base size of $\Delta x=0.08 \mathrm{~mm}$ was chosen leading to 1.2 to 3.1 million polyhedral and prism elements in total depending on the considered vasculature. At each inlet, a time-dependent flow waveform was applied obtained from PC-MRI measurements in a healthy volunteer [26]. Flow-splitting at all outlet cross-sections was defined according to the recent model by Chnafa et al. [27]. Blood was treated as an incompressible $\left(\rho=1055 \mathrm{~kg} / \mathrm{m}^{3}\right)$ and Newtonian ( $\eta=4 \mathrm{mPa} \mathrm{s}$ ) fluid, while the flow was assumed to be laminar. Three cardiac cycles were simulated, while only the last one was considered for analysis.

Quantification of shear-related parameters was conducted for enhanced and non-enhanced wall segments separately, including time-averaged wall shear stress (AWSS), normalized wall shear stress (nWSS), maximum oscillatory shear index (maxOSI), and low shear area (LSA). Mean neck inflow rate (meanNIR), inflow concentration index (ICI), and shear concentration index (SCI) were determined for entire aneurysms [28].

\section{Semi-automatic extraction of morphological parameters}

In each aneurysm, a semiautomatic neck curve reconstruction was performed. First, four points in the neck region of the aneurysm were determined following a manual one-click selection of the aneurysm. Based on these points, a neck curve
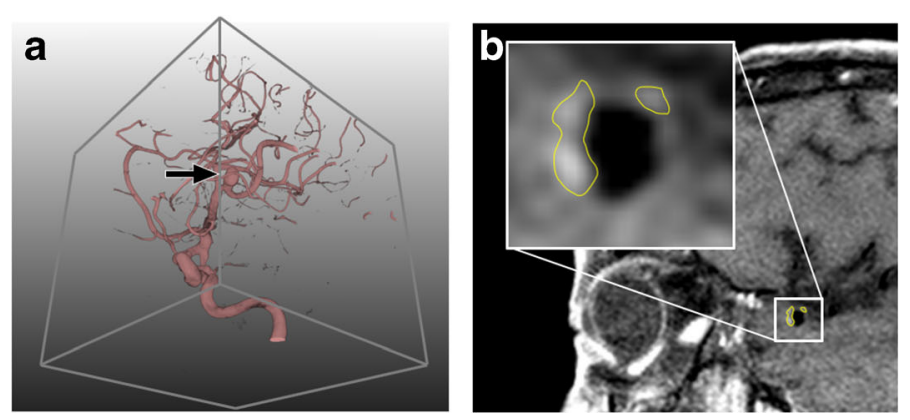

Fig. 1 A Illustration of a 3D rotational angiography (3D-RA) dataset of a middle cerebral artery aneurysm (arrow). B Corresponding MRI dataset with yellow contours delineating enhanced regions. C 3D model of the was created and the ostium plane derived. For a detailed description of the computational process, see Saalfeld et al. [29]. Utilizing the neck curve and ostium plane, the following morphological parameters were automatically extracted: aneurysm surface area (A), aneurysm volume $(\mathrm{V})$, ostium area1 (OA1), ostium area2 (OA2), maximum diameter (Dmax), maximum height (Hmax), maximum width perpendicular to Hmax (Wmax), maximum height perpendicular to the ostium plane (Hortho), maximum width parallel to the ostium plane (Wortho), maximum neck curve diameter (Nmax), average neck curve diameter (Navg), aspect ratio 1 (AR1), aspect ratio 2 (AR2), volume of the convex hull (Vch), surface area of the convex hull (Ach), ellipticity index (EI), non-sphericity index (NSI), undulation index (UI), and aneurysm tilt angle $\gamma$ [29].

\section{Aneurysm clipping and histologic analysis}

Microsurgical clipping of the aneurysms was performed via a pterional-transsylvian approach.

Preoperative, postoperative, and intraoperative management of the patients was performed according to institutional standards, including preoperative and postoperative DSA of the intracranial vessels, and, in most cases, intraoperative angiography with indocyanine green. After clipping of the aneurysm, the aneurysm sac was removed.

Histologic analysis was performed as described before [30]. In brief, formalin-fixed and paraffin-embedded resection specimens were cut into $2.5-\mu \mathrm{m}$-thin tissue sections. Slides were stained either with hematoxylin and eosin, or using rabbit anti-MPO polyclonal antibodies (1:1000, DAKO, Glostrup, Denmark) after antigen retrieval with ER2 (EDTA-buffer bond $\mathrm{pH}$ 8.9), or a monoclonal antibody against CD34 (Beckman Coulter, Clone QBEnd10, 1:700) without antigen retrieval using the autostainer Bond Max System (Leica Microsystems GmbH, Wetzlar, Germany).

\section{Statistical analysis}

Statistical analysis was performed in RStudio (RStudio v1.2.1335 with R v3.5.1, RStudio Inc., Boston, USA).
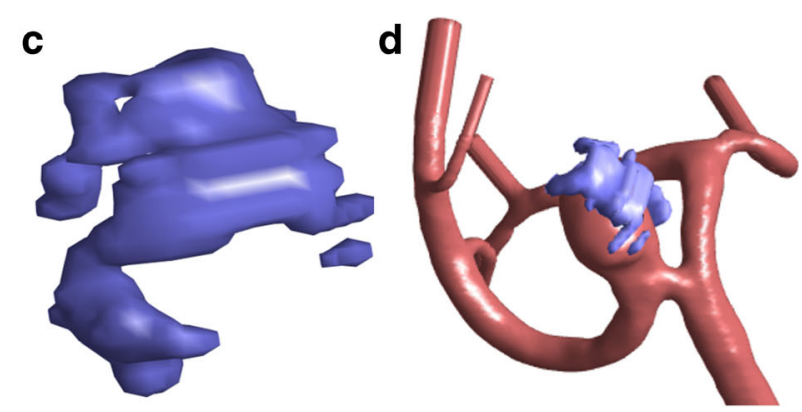

enhanced regions. D Co-registered 3D-RA aneurysm surface mesh and 3D MRI enhancement surface mesh 
Normally distributed variables are presented as mean (range), non-normally distributed continuous variables as median (range) or median (standard deviation), and categorical variables in the form of percentage distributions. A Shapiro-Wilk test was employed to determine, if a continuous variable was normally distributed. Non-normally distributed data were nonlinearly transformed before they were included in univariate analysis. Hemodynamic parameters were analyzed using the Wilcoxon signed-rank test for comparison of enhanced regions and the entire aneurysm surface, and the MannWhitney $U$ test for comparison of the enhancement area EA in aneurysms with and without histologic markers of inflammation and wall degeneration. Pearson's correlation test was used to assess the relationship of hemodynamic and morphological parameters with the enhancement area EA. Only parameters that were significantly associated with EA in univariate analysis were entered into a linear regression model in order to restrict the number of variables included in multivariate analysis. Furthermore, since some of the morphological variables have been reported to show a strong degree of correlation [29], only the variable with the lowest $P$ value was selected for multivariate analysis. A $P<0.05$ was considered significant in univariate and multivariate analyses.

\section{Results}

Twenty-one patients with 22 aneurysms were included; $71 \%$ were located on the right side. Clinical data were available for all included patients. The interrater agreement concerning focal enhancement was rated as substantial (Cohen's $\kappa=0.742$ ). Demographic information of the patients is summarized in Table 1. The PHASES score was positively correlated with EA $(P=0.0275)$. An association of gender, age, hypertension, diabetes, ASA and statin intake, smoking, and aneurysm location with EA could not be demonstrated.

Table 1 Demographic characteristics

\section{Parameter}

\begin{tabular}{ll}
\hline Gender ratio (\% of female) & $76 \%$ \\
Age (mean (range)) & 60 years (48-84) \\
Hypertension & $86 \%$ \\
Diabetes & $14 \%$ \\
ASA & $24 \%$ \\
Statin & $14 \%$ \\
Nicotine & $57 \%$ \\
PHASES (median (mode)) & $6(6)$ \\
\hline
\end{tabular}

Demographic data of all included patients

ASA acetylsalicylic acid, PHASES population, hypertension, age, size of aneurysm, earlier subarachnoid hemorrhage from another aneurysm, site of aneurysm
Microsurgical clipping with subsequent histologic analysis of the resected aneurysm sac was conducted in 9 patients. The surgical indication was based on the patient's preference in 5 cases, and on aneurysm morphology (wide-necked aneurysm) in 4. Features of all aneurysms alongside histologic data are listed in Table 2.

\section{Wall enhancement and blood flow evaluation}

Flow simulation and calculation of hemodynamic variables were successful in all patients. A qualitative observation of the blood flow revealed that flow structures perpendicular to the main flow direction occurred in the vicinity of the enhancement zones. In cases with an increased aspect ratio, it was observed that these flows move sideward through the aneurysm sac instead of following the global flow direction. Additionally, it is important to highlight that enhancement appeared predominantly at the sides of the wall and was only present at the aneurysm dome in one case. Thus, the entering flow-jet and the corresponding impingement zone (which causes increased shear stresses on the luminal surface) were not associated with the enhanced wall segments.

To further quantify the flow behavior along the aneurysmal lumen comparing enhanced and non-enhanced regions, three shear-related parameters were assessed.

AWSS was significantly lower in enhanced wall segments (3.74 Pa (3.91 Pa)) compared with the entire aneurysm wall (7.76 Pa $(4.78 \mathrm{~Pa}))(P<0.0001)$, while maxOSI decreased from $0.45(0.05)$ to $0.37(0.14)(P<0.0001)$. In the analysis of LSA, values were significantly higher in enhanced segments $(68.09 \%(34.36 \%))$ compared with the entire aneurysm $(56.91 \%(30.34 \%))(P<0.0001)$.

There was no significant correlation of inflow-related hemodynamic parameters or SCI with EA. There was a trend towards higher LSA in aneurysms with a larger extent of enhancement $(P=0.08222)$. For a summary of the analysis of hemodynamic and morphologic parameters, see Table 3.

\section{Wall enhancement and morphologic parameters}

OA2, Dmax, Hmax, Wortho, Vch, Ach, EI, NSI, and UI were significantly correlated with EA in univariate analysis (Table 3). EI showed the lowest $P$ value and was entered alongside Dmax into multiple regression analysis, the remaining variables were not considered due to collinearity as described before [29]. EI remained significantly associated with EA $(P=0.0395)$.

\section{Histologic features}

Four aneurysms stained positive for MPO-expressing cells. In three aneurysms, vasa vasorum were present. CD34-positive neovessel endothelium could not be detected in any of the 
Table 2 Size, enhancement area, and histologic features of all aneurysms

\begin{tabular}{|c|c|c|c|c|c|c|c|}
\hline \multirow{2}{*}{$\begin{array}{l}\text { Aneurysm } \\
\text { no. }\end{array}$} & \multirow{2}{*}{$\begin{array}{l}\text { Size } \\
(\mathrm{mm})\end{array}$} & \multirow{2}{*}{$\begin{array}{l}\text { Enhancement } \\
\text { area }(\%)\end{array}$} & \multicolumn{5}{|c|}{ Histologic parameter } \\
\hline & & & MPO & CD34 & $\begin{array}{l}\text { Vasa } \\
\text { vasorum }\end{array}$ & $\begin{array}{l}\text { Lipid } \\
\text { deposition }\end{array}$ & Thrombus \\
\hline 1 & 8 & 2.13 & & & & & \\
\hline 2 & 9 & 0.97 & & & & & \\
\hline 3 & 11 & 22.51 & + & & & + & + \\
\hline 4 & 9 & 6.49 & + & & + & + & \\
\hline 5 & 7 & 31.39 & + & & & & \\
\hline 6 & 7 & 0.49 & & & & & \\
\hline 7 & 10 & 43.09 & + & & + & & + \\
\hline 8 & 6 & 7.30 & & & & & \\
\hline 9 & 5 & 33.23 & & & + & & \\
\hline 10 & 9 & 32.79 & & & & & \\
\hline 11 & 8 & 28.27 & & & & & \\
\hline 12 & 11 & 73.64 & & & & & \\
\hline 13 & 7 & 29.79 & & & & & \\
\hline 14 & 8 & 26.55 & & & & & \\
\hline 15 & 3 & 32.40 & & & & & \\
\hline 16 & 15 & 58.60 & & & & & \\
\hline 17 & 23 & 23.40 & & & & & \\
\hline 18 & 8 & 5.43 & & & & & \\
\hline 19 & 5 & 41.74 & & & & & \\
\hline 20 & 7 & 33.65 & & & & & \\
\hline 21 & 8 & 25.22 & & & & & \\
\hline 22 & 7 & 30.11 & & & & & \\
\hline Median & 8 & 26.55 & & & & & \\
\hline
\end{tabular}

Size (as manually measured on 2D angiographic images), enhancement area, and histologic features of all aneurysm. Histologic specimens were available from aneurysms 1-9 aneurysms. Representative cases are illustrated in Figs. 2 and 3. In this subset of patients, the presence of one or two of these histologic markers was significantly associated with a larger extent of wall enhancement $(P=0.03175)$.

Table $3 \quad P$ values from univariate analyses of the relationship of enhancement (enhancement area EA) with hemodynamic and morphologic parameters

\begin{tabular}{|c|c|c|c|}
\hline Hemodynamic parameter & $P$ value & Morphologic parameter & $P$ value \\
\hline Time-averaged wall shear stress (AWSS) & 0.5696 & Aneurysm surface area (A) & 0.06235 \\
\hline Normalized WSS (nWSS) & 0.8343 & Aneurysm volume (V) & 0.06894 \\
\hline Maximum oscillatory shear index (maxOSI) & 0.217 & Ostium area1 (OA1) & 0.06962 \\
\hline Low shear area (LSA) & 0.08222 & Ostium area2 (OA2) & $0.04929 *$ \\
\hline Mean neck inflow rate (meanNIR) & 0.9488 & Maximum diameter (Dmax) & $0.02093 *$ \\
\hline Inflow concentration index (ICI) & 0.1237 & Maximum height (Hmax) & $0.0421 *$ \\
\hline \multirow[t]{13}{*}{ Shear concentration index (SCI) } & 0.6168 & Maximum width (Wmax) & 0.1363 \\
\hline & & Maximum height perpendicular to the ostium plane (Hortho) & 0.1349 \\
\hline & & Maximum width parallel to the ostium plane (Wortho) & $0.04025^{*}$ \\
\hline & & Maximum neck curve diameter (Nmax) & 0.2294 \\
\hline & & Average neck curve diameter (Navg) & 0.1107 \\
\hline & & Aspect ratio 1 (AR1) & 0.7308 \\
\hline & & Aspect ratio 2 (AR2) & 0.8133 \\
\hline & & Volume of the convex hull (Vch) & $0.04633^{*}$ \\
\hline & & Surface area of the convex hull (Ach) & $0.04644 *$ \\
\hline & & Ellipticity index (EI) & $0.008209^{*}$ \\
\hline & & Non-sphericity index (NSI) & $0.04134 *$ \\
\hline & & Undulation index (UI) & $0.03966^{*}$ \\
\hline & & Aneurysm tilt angle $\gamma$ & 0.7401 \\
\hline
\end{tabular}

*Statistically significant $(P<0.05)$ 

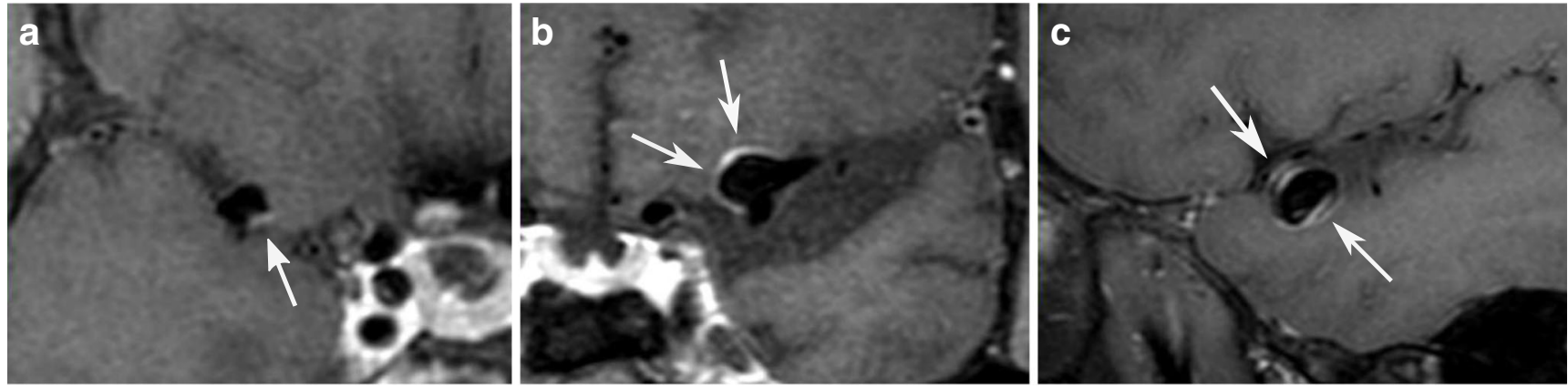

Fig. 2 Post-contrast 3D T1 black blood images showing A aneurysm with a low extent of wall enhancement and B aneurysm with substantial enhancement of the wall. C Double layer appearance in an aneurysm with wall-adjacent thrombus that was histologically confirmed

Wall-adherent thrombus was detected on MR vessel wall imaging in two aneurysms, and intraluminal wall adherent thrombus was confirmed by histologic analysis. Enhancement was visible both on the luminal surface of the thrombus and the adjacent wall, leading to a double-layer appearance (Fig. 2c).

Extracellular lipid deposition was detected in two aneurysms. Hyalinosis, fibrosis, and calcification were common features in most aneurysms.

\section{Discussion}

\section{Focal enhancement, hemodynamic parameters, and morphology}

In this study, focal enhancement was colocalized with low AWSS, low maxOSI, and high LSA in agreement with results recently published by Xiao et al. and Khan et al. [13, 31], confirming the assumption that enhanced regions possibly indicate the presence and localization of low-flow conditions in an aneurysm.
This observation adds to the assumption that the extent of focal enhancement depends on the prevalence of low-flow conditions. Furthermore, it seems likely that there is a continuous spectrum of the extent of wall enhancement correlated to a hemodynamic environment promoting wall destabilization that has to be considered when assessing rupture risk, rather than an either-or presumption that is implied when dichotomizing unruptured aneurysms into either enhancing (and thus at higher rupture risk) or non-enhancing. There was no significant correlation of AWSS, maxOSI, and LSA with EA when computed for whole aneurysms. Still, there was a trend towards higher LSA in aneurysms with a larger extent of enhancement $(P=0.08222)$. This could be explained by the fact that the percentage of the aneurysm surface that was covered by enhancement was relatively low in the aneurysms included in this study (median 27\%; see Table 3). Future longitudinal studies focusing on quantification of aneurysm wall enhancement on MR vessel wall imaging and correlated clinical parameters are warranted.

Numerous studies have described morphologic characteristics associated with a ruptured state, such as larger aneurysm size and greater aspect ratio [15, 17, 22, 32, 33]. Yet, a retrospective longitudinal analysis conducted by Leemans et al.
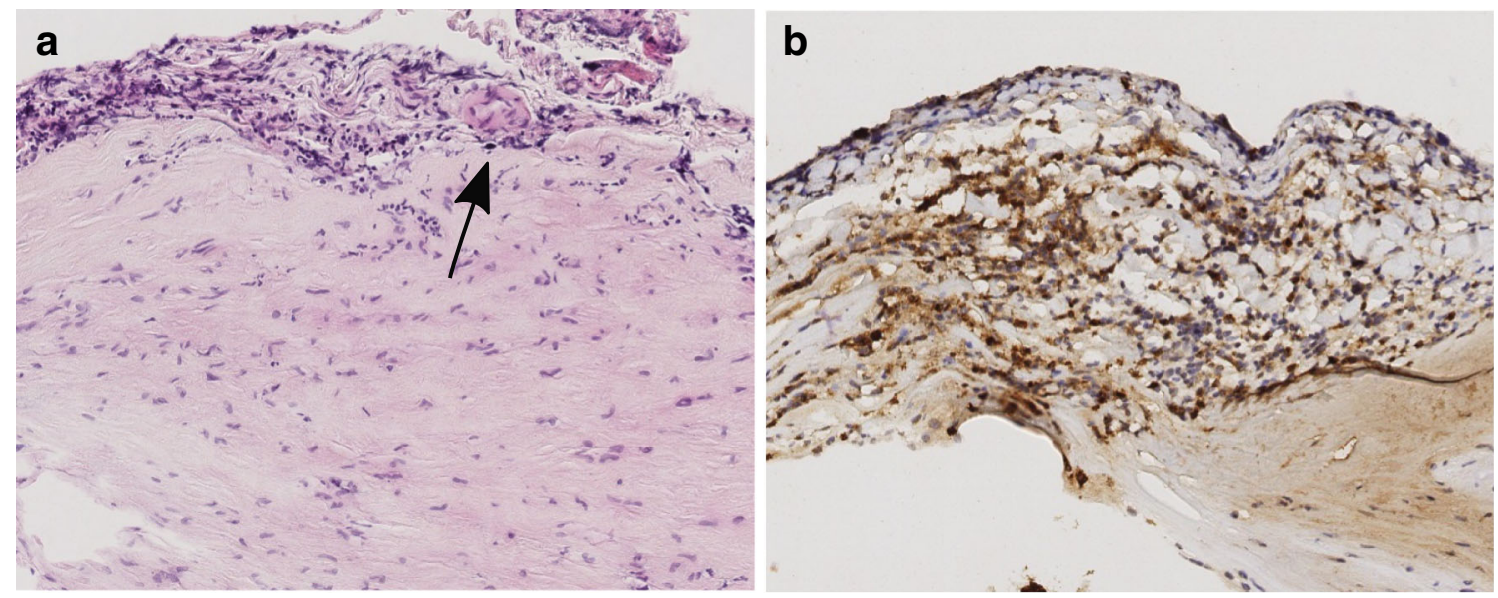

Fig. 3 Representative photomicrographs showing A vasa vasorum (arrow) in the adventitial layer (hematoxylin and eosin stain, original magnification $\times$ 10 ), and $\mathbf{B}$ numerous myeloperoxidase-expressing cells staining brown (myeloperoxidase stain, $\times 10$ ) 
yielded no significant differences in hemodynamic and morphological parameters at baseline imaging in aneurysms that had grown versus stable aneurysms [23]. Morphological and hemodynamic analysis in cases where longitudinal data are not available might therefore not be able to sufficiently discriminate between stable and unstable aneurysms. In our patient cohort, aneurysm enhancement was positively correlated with hemodynamic, morphologic, and histologic markers that have been linked to an unstable state, which supports the assumption that wall enhancement might reflect the complex interactions and multifactorial pathologic processes in aneurysm evolution leading to wall destabilization, while a direct causal relation to either of these factors cannot readily be assumed.

\section{Low-flow conditions, thrombi, and histologic findings}

In a comprehensive study linking macroscopic wall appearances to specific hemodynamic conditions, Cebral et al. found low AWSS to be associated with macroscopically hyperplastic and atherosclerotic wall segments [34]. Concordantly, we observed histologic markers of inflammatory and remodeling processes in aneurysms with a greater extent of focal wall enhancement, suggesting that these pathologic conditions are more abundant in conjunction with low-flow conditions.

Moreover, rupture sites of intracranial aneurysms have been associated with low wall shear stress $[14,34-36]$ and focal enhancement $[11,12]$. Still, a direct association of lowflow areas and possible future rupture site cannot directly be deduced from the results of the present study, because local matching of histologic findings and enhancements areas was not conducted.

Recently, Sato et al. published their results from a 7T vessel wall MRI study describing the double-layer appearance in thrombosed aneurysms and linking them to histologic signs of inflammation, in accordance with our results [37].

Matsushige et al. described focal enhancement as possibly attributable to the presence of thrombotic material at rupture sites [11]. Another study attempted to categorize aneurysm wall types and found thrombosis as a feature of categories with a higher proportion of ruptured aneurysms [38]. These observations add to the assumption that slow blood flow and thrombus formation might play a substantial role in wall destabilization and can be visualized with MR vessel wall imaging.

Still, the observation that focal enhancement, albeit to a lesser extent, is also encountered in aneurysms lacking histologic signs of wall inflammation, possibly reflects the proposed theory that there are different pathways of wall destabilization in intracranial aneurysms [39]. Substantial wall enhancement might primarily reflect the inflammatory pathway of wall remodeling, while the sensitivity for the mural-cellmediated pathway could be diminished.

Cornelissen et al. assumed that wall enhancement is not causally linked with inflammatory processes but rather attributable to method-inherent failure of blood signal suppression in low-flow conditions [40, 41]. The results of our study suggest that inflammatory changes probably add to the extent of wall enhancement, and that even without convincing proof of a causal link, wall enhancement indicates the presence of a hemodynamic environment promoting inflammatory and degenerative processes and might therefore still serve as a surrogate marker for aneurysm wall instability.

The extent of enhancement seems to depend on the sequence employed in the vessel wall MR protocol, with the T1 TSE black blood sequence like the one used in our study possibly being more sensitive for the detection of slow blood flow compared with motion-sensitized driven equilibrium (MSDE)-prepared sequences [42].

This study has several limitations. The retrospective study design might imply a selection bias towards patients who underwent DSA and were potentially pre-selected for therapy due to their risk profiles. Additionally, histologic analysis was exclusively performed in patients who underwent clipping, possibly inducing bias towards aneurysms with morphologies unfavorable for endovascular therapy. Histological data were only available in nine aneurysms. Microsurgical clipping provides only specimens of the aneurysm dome; neck segments under the clip cannot be retrieved. The small sample size precluded subgroup analysis of histologic features with adequate power. We chose to restrict our analysis to middle cerebral artery aneurysms in an effort to exclude a possible heterogeneity in aneurysm phenotype caused by different pathogenetic mechanisms potentially underlying the evolution and progression of aneurysms in different locations [43]. Additionally, an overestimation of the neck size using 3D imaging techniques can occur compared with 2D images, but careful segmentation using advanced algorithms was applied [44, 45]. Finally, no patient-specific inflow conditions were available for the blood flow simulations. However, a comparison between enhanced regions and the whole aneurysm remains feasible.

\section{Conclusion}

Focal wall enhancement in unruptured intracranial aneurysms is colocalized with hemodynamic factors that have been related to a higher risk of rupture. It is correlated with morphological factors linked to rupture risk, a higher PHASES score, and histologic markers of wall destabilization. The results support the hypothesis that focal enhancement could serve as a surrogate marker for aneurysm instability.

Acknowledgments Open Access funding provided by Projekt DEAL.

Funding information This study was funded by the Federal Ministry of Education and Research in Germany within the Forschungscampus STIMULATE (grant number 13GW0095A), the German Research 
Foundation (grant number SA 3461/2-1, BE 6230/2-1), and the European Regional Development Fund (ZS/2016/04/78123).

Data availability The datasets analyzed in the current study are available from the corresponding author on reasonable request.

\section{Compliance with ethical standards}

Conflict of interest The authors declare that they have no conflict of interest.

Ethical approval All procedures performed in studies involving human participants were in accordance with the ethical standards of the institutional and/or national research committee and with the 1964 Helsinki declaration and its later amendments or comparable ethical standards.

Approval was obtained from the Ethics Committee of the ChristianAlbrechts-University Kiel, Germany.

This article does not contain any studies with animals performed by any of the authors.

Informed consent Informed consent was obtained from all individual participants included in the study.

Code availability Not applicable.

Open Access This article is licensed under a Creative Commons Attribution 4.0 International License, which permits use, sharing, adaptation, distribution and reproduction in any medium or format, as long as you give appropriate credit to the original author(s) and the source, provide a link to the Creative Commons licence, and indicate if changes were made. The images or other third party material in this article are included in the article's Creative Commons licence, unless indicated otherwise in a credit line to the material. If material is not included in the article's Creative Commons licence and your intended use is not permitted by statutory regulation or exceeds the permitted use, you will need to obtain permission directly from the copyright holder. To view a copy of this licence, visit http://creativecommons.org/licenses/by/4.0/.

\section{References}

1. Etminan N, Rinkel GJ (2016) Unruptured intracranial aneurysms: development, rupture and preventive management. Nat Rev Neurol 12:699-713

2. Wiebers DO, Whisnant JP, Huston J, Meissner I, Brown RD, Piepgras DG et al (2003) Unruptured intracranial aneurysms: natural history, clinical outcome, and risks of surgical and endovascular treatment. Lancet 362:103-110

3. Nieuwkamp DJ, Setz LE, Algra A, Linn FHH, de Rooij NK, Rinkel GJE (2009) Changes in case fatality of aneurysmal subarachnoid haemorrhage over time, according to age, sex, and region: a metaanalysis. Lancet Neurol 8:635-642

4. Edjlali M, Guédon A, Ben Hassen W, Boulouis G, Benzakoun J, Rodriguez-Régent C, Trystram D, Nataf F, Meder JF, Turski P, Oppenheim C, Naggara O (2018) Circumferential thick enhancement at vessel wall MRI has high specificity for intracranial aneurysm instability. Radiology 289:181-187

5. Hartman JB, Watase H, Sun J, Hippe DS, Kim L, Levitt M, Sekhar L, Balu N, Hatsukami T, Yuan C, Mossa-Basha M (2019) Intracranial aneurysms at higher clinical risk for rupture demonstrate increased wall enhancement and thinning on multi-contrast 3d vessel wall MRI. Br J Radiol 92:20180950. https://doi.org/10. 1259/bjr.20180950

6. Lv N, Karmonik C, Chen S, Wang X, Fang Y, Huang Q, Liu J (2019) Relationship between aneurysm wall enhancement in vessel wall magnetic resonance imaging and rupture risk of unruptured intracranial aneurysms. Neurosurgery 84:E385-E391. https://doi. org/10.1093/neuros/nyy310

7. Hu P, Yang Q, Wang D-D, Guan S-C, Zhang H-Q (2016) Wall enhancement on high-resolution magnetic resonance imaging may predict an unsteady state of an intracranial saccular aneurysm. Neuroradiology 58:979-985

8. Matouk CC, Mandell DM, Günel M, Bulsara KR, Malhotra A, Hebert R, Johnson MH, Mikulis DJ, Minja FJ (2013) Vessel wall magnetic resonance imaging identifies the site of rupture in patients with multiple intracranial aneurysms: proof of principle. Neurosurgery 72:492-496

9. Bhogal P, Lansley J, Wong K, Udani SD, Uff C, Wadley J, Kumar A, Matouk CC, Makalanda HLD (2019) Vessel wall enhancement of a ruptured intra-nidal aneurysm in a brain arteriovenous malformation. Interv Neuroradiol 25:310-314

10. Larsen N, von der Brelie C, Trick D, Riedel CH, Lindner T, Madjidyar J, Jansen O, Synowitz M, Flüh C (2018) Vessel wall enhancement in unruptured intracranial aneurysms: an Indicator for higher risk of rupture? High-resolution MR imaging and correlated histologic findings. Am J Neuroradiol 39:1617-1621

11. Matsushige T, Shimonaga K, Mizoue T, Hosogai M, Hashimoto Y, Kaneko M, Ono C, Ishii D, Sakamoto S, Kurisu K (2019) Focal aneurysm wall enhancement on magnetic resonance imaging indicates intraluminal thrombus and the rupture point. World Neurosurg 127:e578-e584

12. Wang G, Wen L, Lei S, Ran Q, Yin J, Gong Z et al (2018) Wall enhancement ratio and partial wall enhancement on MRI associated with the rupture of intracranial aneurysms. J Neurointerv Surg 10: 566-570

13. Xiao W, Qi T, He S, Li Z, Ou S, Zhang G, Liu X, Huang Z, Liang F (2018) Low wall shear stress is associated with local aneurysm wall enhancement on high-resolution MR vessel wall imaging. Am J Neuroradiol 39:2082-2087

14. Omodaka S, Sugiyama S-I, Inoue T, Funamoto K, Fujimura M, Shimizu H, Hayase T, Takahashi A, Tominaga T (2012) Local hemodynamics at the rupture point of cerebral aneurysms determined by computational fluid dynamics analysis. Cerebrovasc Dis 34:121-129

15. Chung BJ, Mut F, Putman CM, Hamzei-Sichani F, Brinjiki W, Kallmes D et al (2018) Identification of hostile hemodynamics and geometries of cerebral aneurysms: a case-control study. Am J Neuroradiol 39:1860-1866

16. Berg P, Beuing O (2018) Multiple intracranial aneurysms: a direct hemodynamic comparison between ruptured and unruptured vessel malformations. Int J CARS 13:83-93

17. Xiang J, Natarajan SK, Tremmel M, Ma D, Mocco J, Hopkins LN, Siddiqui AH, Levy EI, Meng H (2011) Hemodynamicmorphologic discriminants for intracranial aneurysm rupture. Stroke 42:144-152

18. Xiang J, Yu J, Snyder KV, Levy EI, Siddiqui AH, Meng H (2016) Hemodynamic-morphological discriminant models for intracranial aneurysm rupture remain stable with increasing sample size. J Neurointerv Surg 8:104-110

19. Liang L, Steinman DA, Brina O, Chnafa C, Cancelliere NM, Pereira VM (2019) Towards the clinical utility of CFD for assessment of intracranial aneurysm rupture - a systematic review and novel parameter-ranking tool. J Neurointerv Surg 11:153-158

20. Zhang Y, Jing L, Liu J, Li C, Fan J, Wang S, Li H, Yang X (2016) Clinical, morphological, and hemodynamic independent characteristic factors for rupture of posterior communicating artery aneurysms. J Neurointerv Surg 8:808-812 
21. Jing L, Fan J, Wang Y, Li H, Wang S, Yang X, Zhang Y (2015) Morphologic and hemodynamic analysis in the patients with multiple intracranial aneurysms: ruptured versus unruptured. PLoS One 10:e132494

22. Dhar S, Tremmel M, Mocco J, Kim M, Yamamoto J, Siddiqui AH, Hopkins LN, Meng H (2008) Morphology parameters for intracranial aneurysm rupture risk assessment. Neurosurgery 63:185-196

23. Leemans EL, Cornelissen BMW, Slump CH, Majoie CBLM, Cebral JR, Marquering HA (2019) Comparing morphology and hemodynamics of stable-versus-growing and grown intracranial aneurysms. Am J Neuroradiol 40:2102-2110

24. Leemans EL, Cornelissen BMW, Said M, van den Berg R, Slump $\mathrm{CH}$, Marquering HA, Majoie CBLM (2019) Intracranial aneurysm growth: consistency of morphological changes. Neurosurg Focus 47:E5

25. Haber E, Modersitzki J (2006) Intensity gradient based registration and fusion of multi-modal images. Lect Notes Comput Sc 9:726733

26. Berg P, Stucht D, Janiga G, Beuing O, Speck O, Thévenin D (2014) Cerebral blood flow in a healthy Circle of Willis and two intracranial aneurysms: computational fluid dynamics versus fourdimensional phase-contrast magnetic resonance imaging. $\mathrm{J}$ Biomech Eng 136(4). https://doi.org/10.1115/1.4026108

27. Chnafa C, Brina O, Pereira VM, Steinman DA (2018) Better than nothing: a rational approach for minimizing the impact of outflow strategy on cerebrovascular simulations. Am J Neuroradiol 39:337343

28. Cebral JR, Mut F, Weir J, Putman C (2011) Quantitative characterization of the hemodynamic environment in ruptured and unruptured brain aneurysms. Am J Neuroradiol 32:145-151

29. Saalfeld S, Berg P, Niemann A, Luz M, Preim B, Beuing O (2018) Semiautomatic neck curve reconstruction for intracranial aneurysm rupture risk assessment based on morphological parameters. Int $\mathbf{J}$ Comput Ass Rad 13:1781-1793

30. Warneke VS, Behrens H-M, Haag J, Balschun K, Böger C, Becker T, Ebert MPA, Lordick F, Röcken C (2013) Prognostic and putative predictive biomarkers of gastric cancer for personalized medicine. Diagn Mol Pathol 22:127-137

31. Khan MO, Toro Arana V, Rubbert C, Cornelius JF, Fischer I, Bostelmann R, Mijderwijk HJ, Turowski B, Steiger HJ, May R, Petridis AK (2020) Association between aneurysm hemodynamics and wall enhancement on 3D vessel wall MRI. J Neurosurg:1-11. https://doi.org/10.3171/2019.10.JNS191251

32. Detmer FJ, Chung BJ, Jimenez C, Hamzei-Sichani F, Kallmes D, Putman C, Cebral JR (2019) Associations of hemodynamics, morphology, and patient characteristics with aneurysm rupture stratified by aneurysm location. Neuroradiology 61:275-284

33. Xu L, Wang H, Chen Y, Dai Y, Lin B, Liang F et al (2020) Morphological and hemodynamic factors associated with ruptured middle cerebral artery mirror Aneurysms: A retrospective study. World Neurosurg. https://doi.org/10.1016/j.wneu.2020.01.083

34. Cebral JR, Detmer F, Chung BJ, Choque-Velasquez J, Rezai B, Lehto H, Tulamo R, Hernesniemi J, Niemela M, Yu A, Williamson R, Aziz K, Shakur S, Amin-Hanjani S, Charbel F, Tobe Y, Robertson A, Frösen J (2019) Local hemodynamic conditions associated with focal changes in the intracranial aneurysm wall. Am J Neuroradiol 40:510-516

35. Fukazawa K, Ishida F, Umeda Y, Miura Y, Shimosaka S, Matsushima S, Taki W, Suzuki H (2015) Using computational fluid dynamics analysis to characterize local hemodynamic features of middle cerebral artery aneurysm rupture points. World Neurosurg 83:80-86

36. Suzuki T, Stapleton CJ, Koch MJ, Tanaka K, Fujimura S, Suzuki T et al (2019) Decreased wall shear stress at high-pressure areas predicts the rupture point in ruptured intracranial aneurysms. J Neurosurg 15:1-7

37. Sato T, Matsushige T, Chen B, Gembruch O, Dammann P, Jabbarli R, Forsting M, Junker A, Maderwald S, Quick HH, Ladd ME, Sure U, Wrede KH (2019) Wall contrast enhancement of thrombosed intracranial aneurysms at 7T MRI. Am J Neuroradiol 40:11061111

38. Frösen J, Piippo A, Paetau A, Kangasniemi M, Niemelä M, Hernesniemi J et al (2004) Remodeling of saccular cerebral artery aneurysm wall is associated with rupture: histological analysis of 24 unruptured and 42 ruptured cases. Stroke 35:2287-9339

39. Meng H, Tutino VM, Xiang J, Siddiqui A (2014) High WSS or low WSS? Complex interactions of hemodynamics with intracranial aneurysm initiation, growth, and rupture: toward a unifying hypothesis. Am J Neuroradiol 35:1254-1262

40. Cornelissen BMW, Leemans EL, Coolen BF, Peper ES, van den Berg R, Marquering HA, Slump CH, Majoie CBLM (2019) Insufficient slow-flow suppression mimicking aneurysm wall enhancement in magnetic resonance vessel wall imaging: a phantom study. Neurosurg Focus 47:E19

41. Cornelissen BMW, Leemans EL, Slump CH, Marquering HA, Majoie CBLM, van den Berg R (2019) Vessel wall enhancement of intracranial aneurysms: fact or artifact? Neurosurg Focus 47:E18

42. Kalsoum E, Negrier AC, Tuilier T, Benaïssa A, Blanc R, Gallas S et al (2018) Blood flow mimicking aneurysmal Wall enhancement: a diagnostic pitfall of vessel wall MRI using the postcontrast 3D turbo spin-echo MR imaging sequence. Am J Neuroradiol 39: 1065-1067

43. Varble N, Rajabzadeh-Oghaz H, Wang J, Siddiqui A, Meng H, Mowla A (2017) Differences in morphologic and hemodynamic characteristics for "PHASES-based" intracranial aneurysm locations. Am J Neuroradiol 38:2105-2110

44. Berg P, Saalfeld S, Voß S, Redel T, Preim B, Janiga G, Beuing O (2018) Does the DSA reconstruction kernel affect hemodynamic predictions in intracranial aneurysms? An analysis of geometry and blood flow variations. J Neurointerv Surg 10:290-296

45. Berg P, Voß S, Saalfeld S, Janiga G, Bergersen AW, ValenSendstad K, Bruening J, Goubergrits L, Spuler A, Cancelliere NM, Steinman DA, Pereira VM, Chiu TL, Tsang ACO, Chung BJ, Cebral JR, Cito S, Pallarès J, Copelli G, Csippa B, Paál G, Fujimura S, Takao H, Hodis S, Hille G, Karmonik C, Elias S, Kellermann K, Khan MO, Marsden AL, Morales HG, Piskin S, Finol EA, Pravdivtseva M, Rajabzadeh-Oghaz H, Paliwal N, Meng H, Seshadhri S, Howard M, Shojima M, Sugiyama SI, Niizuma K, Sindeev S, Frolov S, Wagner T, Brawanski A, Qian Y, Wu YA, Carlson KD, Dragomir-Daescu D, Beuing O (2018) Multiple Aneurysms AnaTomy CHallenge 2018 (MATCH): phase I: segmentation. Cardiovasc Eng Technol 9:565-581

Publisher's note Springer Nature remains neutral with regard to jurisdictional claims in published maps and institutional affiliations. 